Kragujevac Journal of Mathematics

Volume 45(2) (2021), Pages 289-298.

\title{
LYAPUNOV-TYPE INEQUALITY FOR AN ANTI-PERIODIC CONFORMABLE BOUNDARY VALUE PROBLEM
}

\author{
JAGAN MOHAN JONNALAGADDA ${ }^{1}$, DEBANANDA BASUA ${ }^{2}$, \\ AND DIPAK KUMAR SATPATHI ${ }^{3}$
}

\begin{abstract}
In this article, we present a Lyapunov-type inequality for a conformable boundary value problem associated with anti-periodic boundary conditions. To demonstrate the applicability of established result, we obtain a lower bound on the eigenvalue of the corresponding eigenvalue problem.
\end{abstract}

\section{INTRODUCTION}

The subject of fractional calculus deals with the theory and applications of integral and differential operators of arbitrary order. The combined efforts of a number of scientists for many years resulted a strong basic theory of fractional calculus $[13,19]$. In this process, several types of fractional differential operators were proposed so far. Unfortunately, each type obeys only some of the properties of the classical derivative.

In 2015, Ortigueira et al. [15] formulated two criteria required by an operator capable of being interpreted as fractional derivative. Recently, Tarasov [20] proposed a principle of nonlocality for fractional derivatives. As a result of these two articles, neither of the conformable differential operators proposed by Khalil et al. [12] are interpreted as fractional derivatives. Further, differential equations with conformable derivatives can be represented as differential equations of integer order for the space of differentiable functions. Subsequently, the conformable derivative was generalized in many ways $[1,10,11]$. Several authors have explored properties [3-7] and physical applications of the conformable derivative [5,6,24]. Recently, Anderson et al. [5]

Key words and phrases. Conformable derivative, boundary value problem, anti-periodic boundary conditions, Green's function, Lyapunov-type inequality, eigenvalue.

2010 Mathematics Subject Classification. Primary: 34A08. Secondary: 26A33, 26 D15.

DOI 10.46793/KgJMat2102.289J

Received: April 27, 2018.

Accepted: December 10, 2018. 
argued that there is a significant value in exploring the mathematics and physical applications of these derivatives.

The Lyapunov inequality is a necessary condition for the existence of a nontrivial solution of Hill's equation associated with Dirichlet boundary conditions.

Theorem 1.1 ([14]). If the boundary value problem

$$
\left\{\begin{array}{l}
y^{\prime \prime}(t)+p(t) y(t)=0, \quad a<t<b \\
y(a)=0, y(b)=0
\end{array}\right.
$$

has a nontrivial solution, where $p:[a, b] \rightarrow \mathbb{R}$ is a continuous function, then

$$
\int_{a}^{b}|p(s)| d s>\frac{4}{(b-a)} .
$$

The Lyapunov inequality (1.2) finds its applications in various problems related to the theory of differential equations and allied fields. Due to its importance, the Lyapunov inequality has been generalized in many forms. For a detailed discussion on Lyapunov-type inequalities and their applications, one can refer $[8,16,18,21-23]$ and the references therein.

On the other hand, Abdeljawad [2] and Gholami et al. [9] independently generalized Theorem 1.1 to the case where the classical second-order derivative in (1.1) is replaced by an $\alpha^{\text {th }}$-order, $1<\alpha \leq 2$, conformable derivative.

Theorem 1.2 ([2]). If the boundary value problem

$$
\left\{\begin{array}{l}
\left(T_{a+}^{\alpha} y\right)(t)+p(t) y(t)=0, \quad a<t<b \\
y(a)=0, \quad y(b)=0
\end{array}\right.
$$

has a nontrivial solution, where $p:[a, b] \rightarrow \mathbb{R}$ is a continuous function, then

$$
\int_{a}^{b}|p(s)| d s>\frac{\alpha^{\alpha}}{(\alpha-1)^{\alpha-1}(b-a)^{\alpha-1}} .
$$

Here $T_{a+}^{\alpha}$ denotes the $\alpha^{\text {th }}$-order conformable differential operator. Motivated by these works, in this article, we derive a Lyapunov-type inequality for the following two-point anti-periodic conformable boundary value problem:

$$
\left\{\begin{array}{l}
\left(T_{0+}^{\alpha} y\right)(t)+p(t) y(t)=0, \quad 1<\alpha \leq 2,0<t<T \\
y(0)+y(T)=0, \quad y^{\prime}(0)+y^{\prime}(T)=0 .
\end{array}\right.
$$

\section{Preliminaries}

Throughout, we shall use the following notations, definitions and known results of conformable calculus $[1,12]$. 
Definition $2.1([1])$. Let $y:[a, \infty) \rightarrow \mathbb{R}$ and $0<\alpha \leq 1$. The $\alpha^{\text {th }}$-order conformable derivative of $y$ starting from $a$ is defined by

$$
\left(T_{a+}^{\alpha} y\right)(t)=\lim _{\varepsilon \rightarrow 0}\left[\frac{y\left(t+\varepsilon(t-a)^{1-\alpha}\right)-y(t)}{\varepsilon}\right], \quad t \in(a, \infty) .
$$

If $\left(T_{a+}^{\alpha} y\right)$ exists on $(a, b)$, then

$$
\left(T_{a+}^{\alpha} y\right)(a)=\lim _{t \rightarrow a^{+}}\left(T_{a+}^{\alpha} y\right)(t) .
$$

Definition 2.2 ([1]). Let $y:[a, \infty) \rightarrow \mathbb{R}, \alpha>0$ and choose $n \in \mathbb{N}_{1}$ such that $n-1<\alpha \leq n$. Assume that $y^{(n-1)}$ exists on $(a, \infty)$. The $\alpha^{\text {th }}$-order conformable derivative of $y$ starting from $a$ is defined by

$$
\begin{aligned}
\left(T_{a+}^{\alpha} y\right)(t) & =\left(T_{a+}^{\alpha-n+1} y^{(n-1)}\right)(t) \\
& =\lim _{\varepsilon \rightarrow 0}\left[\frac{y^{(n-1)}\left(t+\varepsilon(t-a)^{n-\alpha}\right)-y^{(n-1)}(t)}{\varepsilon}\right], \quad t \in(a, \infty) .
\end{aligned}
$$

If $y^{(n)}$ exists on $(a, \infty)$, we have

$$
\left(T_{a+}^{\alpha} y\right)(t)=(t-a)^{n-\alpha} y^{(n)}(t), \quad t \in(a, \infty) .
$$

Also, we define

$$
\left(T_{a+}^{0} y\right)(t)=y(t), \quad t \in(a, \infty) .
$$

Definition $2.3([1])$. Let $y:[a, b] \rightarrow \mathbb{R}, \alpha>0$ and choose $n \in \mathbb{N}_{1}$ such that $n-1<\alpha \leq n$. The $\alpha^{\text {th }}$-order conformable integral of $y$ starting from $a$ is defined by

$$
\left(I_{a+}^{\alpha} y\right)(t)=\frac{1}{(n-1) !} \int_{a}^{t}(t-s)^{n-1}(s-a)^{\alpha-n} y(s) d s, \quad t \in[a, b] .
$$

Theorem $2.1([1])$. Let $y:[a, b] \rightarrow \mathbb{R}, \alpha>0$ and choose $n \in \mathbb{N}_{1}$ such that $n-1<$ $\alpha \leq n$. If $y^{(n-1)}$ exists on $(a, b)$, then

$$
\left(I_{a+}^{\alpha} T_{a+}^{\alpha} y\right)(t)=y(t)-\sum_{k=0}^{n-1} \frac{y^{(k)}(a)(t-a)^{k}}{k !}, \quad t \in(a, b) .
$$

\section{Anti-Periodic Boundary Value Problem}

In this section, we derive a few properties of the Green's function for the boundary value problem (1.3) and obtain the corresponding Lyapunov-type inequality.

Theorem 3.1. Let $1<\alpha \leq 2$ and $h:[0, T] \rightarrow \mathbb{R}$ is a continuous function. The conformal boundary value problem

$$
\left\{\begin{array}{l}
\left(T_{0+}^{\alpha} y\right)(t)+h(t)=0, \quad 0<t<T \\
y(0)+y(T)=0, \quad y^{\prime}(0)+y^{\prime}(T)=0
\end{array}\right.
$$


has the unique solution

where

$$
y(t)=\int_{0}^{T} G(t, s) h(s) d s
$$

$$
G(t, s)= \begin{cases}\left(\frac{T}{4}+\frac{t-s}{2}\right) s^{\alpha-2}, & 0<t \leq s \leq T \\ \left(\frac{T}{4}+\frac{s-t}{2}\right) s^{\alpha-2}, & 0<s \leq t \leq T\end{cases}
$$

Proof. Applying $I_{0+}^{\alpha}$ on both sides of (3.1) and using Theorem 2.1, we have

$$
y(t)=C_{1}+C_{2} t-\int_{0}^{t}(t-s) s^{\alpha-2} h(s) d s .
$$

Differentiating (3.3) with respect to $t$, we get

$$
y^{\prime}(t)=C_{2}-\int_{0}^{t} s^{\alpha-2} h(s) d s .
$$

Using $y(0)+y(T)=0$ in $(3.3)$ we get

$$
2 C_{1}+C_{2} T=\int_{0}^{T}(T-s) s^{\alpha-2} h(s) d s .
$$

Using $y^{\prime}(0)+y^{\prime}(T)=0$ in (3.4) we get

$$
C_{2}=\frac{1}{2} \int_{0}^{T} s^{\alpha-2} h(s) d s .
$$

Then, from (3.5) and (3.6), we have

$$
2 C_{1}=-\frac{T}{2} \int_{0}^{T} s^{\alpha-2} h(s) d s+\int_{0}^{T}(T-s) s^{\alpha-2} h(s) d s,
$$

which implies

$$
C_{1}=\frac{1}{2} \int_{0}^{T}\left(\frac{T}{2}-s\right) s^{\alpha-2} h(s) d s .
$$

Then, from (3.3), (3.6) and (3.7) we have

$$
\begin{aligned}
y(t)= & \frac{1}{2} \int_{0}^{T}\left(\frac{T}{2}-s\right) s^{\alpha-2} h(s) d s+\frac{t}{2} \int_{0}^{T} s^{\alpha-2} h(s) d s-\int_{0}^{t}(t-s) s^{\alpha-2} h(s) d s \\
= & \frac{1}{2} \int_{0}^{t}\left(\frac{T}{2}+t-s\right) s^{\alpha-2} h(s) d s+\frac{1}{2} \int_{t}^{T}\left(\frac{T}{2}+t-s\right) s^{\alpha-2} h(s) d s \\
& -\int_{0}^{t}(t-s) s^{\alpha-2} h(s) d s \\
= & \int_{0}^{t}\left(\frac{T}{4}+\frac{s-t}{2}\right) s^{\alpha-2} h(s) d s+\int_{t}^{T}\left(\frac{T}{4}+\frac{t-s}{2}\right) s^{\alpha-2} h(s) d s \\
= & \int_{0}^{T} G(t, s) h(s) d s .
\end{aligned}
$$

The proof is complete. 
Lemma 3.1. The Green's function $G(t, s)$ defined in (3.2) satisfies the following properties:

(a) $G(t, s) \leq G(s, s),(t, s) \in(0, T] \times(0, T]$;

(b) $s^{2-\alpha} G(s, s)=\frac{T}{4}, s \in[0, T]$;

(c) $\left|s^{2-\alpha} G(t, s)\right| \leq \frac{T}{4},(t, s) \in[0, T] \times[0, T]$;

(d) $\max _{t \in[0, T]} \int_{0}^{T} G(t, s) d s=\frac{T^{\alpha}\left(2-\alpha+2^{\frac{\alpha-2}{\alpha-1}}(\alpha-1)\right)}{4 \alpha(\alpha-1)}$;

(e) $\max _{t \in[0, T]} \int_{0}^{T} s^{2-\alpha} G(t, s) d s=\frac{T^{2}}{8}$;

(f) $\max _{t \in[0, T]} \int_{0}^{T} G^{\prime}(t, s) d s=\frac{T^{\alpha-1}}{2(\alpha-1)}$;

(g) $\max _{t \in[0, T]} \int_{0}^{T} s^{2-\alpha} G^{\prime}(t, s) d s=\frac{T}{2}$;

(h) $\max _{t \in[0, T]} \int_{0}^{T}|G(t, s)| d s=\frac{T^{\alpha}(7 \alpha-2)}{4 \alpha(\alpha-1)}$.

Proof. Define the functions

$$
G_{1}(t, s)=\left(\frac{T}{4}+\frac{t-s}{2}\right) s^{\alpha-2} \quad \text { and } \quad G_{2}(t, s)=\left(\frac{T}{4}+\frac{s-t}{2}\right) s^{\alpha-2} .
$$

We can easily check that $G_{1}(t, s)$ is an increasing function of $t$. Differentiating $G_{2}(t, s)$ with respect to $t$ for every fixed $s$, we observe that, $G_{2}(t, s)$ is a decreasing function of $t$. Thus, we have (a). The proof of (b) follows from (3.2). Clearly, from (a) and (b), we have

$$
s^{2-\alpha} G(t, s) \leq \frac{T}{4}, \quad(t, s) \in[0, T] \times[0, T]
$$

Consider

which implies

$$
s^{2-\alpha} G_{1}(t, s)=\frac{T}{4}+\frac{s-t}{2} \geq \frac{T}{4}+\frac{0-T}{2} \geq-\frac{T}{4},
$$

$$
-s^{2-\alpha} G_{1}(t, s) \leq \frac{T}{4}
$$

Similarly

implies

$$
s^{2-\alpha} G_{2}(t, s)=\frac{T}{4}+\frac{t-s}{2} \geq \frac{T}{4}+\frac{0-T}{2} \geq-\frac{T}{4},
$$

$$
-s^{2-\alpha} G_{2}(t, s) \leq \frac{T}{4} .
$$

So, from (3.9) and (3.10), we get

$$
-s^{2-\alpha} G(t, s) \leq \frac{T}{4}, \quad(t, s) \in[0, T] \times[0, T] .
$$

Then, from (3.8) and (3.11), (c) follows. For (d), consider

$$
\int_{0}^{T} G(t, s) d s=\int_{0}^{t}\left(\frac{T}{4}+\frac{s-t}{2}\right) s^{\alpha-2} d s+\int_{t}^{T}\left(\frac{T}{4}+\frac{t-s}{2}\right) s^{\alpha-2} d s
$$




$$
\begin{aligned}
= & \left(\frac{T}{4}-\frac{t}{2}\right)\left(\frac{t^{\alpha-1}}{\alpha-1}\right)+\frac{t^{\alpha}}{2 \alpha}+\left(\frac{T}{4}+\frac{t}{2}\right)\left[\frac{T^{\alpha-1}}{\alpha-1}-\frac{t^{\alpha-1}}{\alpha-1}\right] \\
& -\frac{1}{2}\left[\frac{T^{\alpha}}{\alpha}-\frac{t^{\alpha}}{\alpha}\right] .
\end{aligned}
$$

Define $H_{1}(t)$ as the right hand side of (3.12). Now, differentiating $H_{1}(t)$ with respect to $t$ and equating it to 0 , we obtain $t=\frac{T}{2^{\frac{1}{\alpha-1}}}$. Again, differentiating $H_{1}{ }^{\prime}(t)$ with respect to $t$, we observe that $H_{1}{ }^{\prime \prime}(t) \leq 0$ at $t=\frac{T}{2^{\frac{1}{\alpha-1}}}$. So, $H_{1}(t)$ attains its maximum at $t=\frac{T}{2^{\frac{1}{\alpha-1}}}$. Thus, we have $(\mathrm{d})$. Consider

$$
\begin{aligned}
\int_{0}^{T} s^{2-\alpha} G(t, s) d s & =\int_{0}^{t}\left(\frac{T}{4}+\frac{s-t}{2}\right) d s+\int_{t}^{T}\left(\frac{T}{4}+\frac{t-s}{2}\right) d s \\
& =\left(\frac{T}{4}-\frac{t}{2}\right) t+\frac{t^{2}}{4}+\left(\frac{T}{4}+\frac{t}{2}\right)(T-t)-\left(\frac{T^{2}-t^{2}}{4}\right) .
\end{aligned}
$$

Define $H_{2}(t)$ as the right hand side of (3.13). Now, differentiating $H_{2}(t)$ with respect to $t$ and equating it to 0 , we obtain $t=\frac{T}{2}$. Again, differentiating $H_{2}{ }^{\prime}(t)$ with respect to $t$, we observe that $H_{2}{ }^{\prime \prime}(t)<0$ at $t=\frac{T}{2}$. So, $H_{2}(t)$ attains its maximum at $t=\frac{T}{2}$. Thus, we have (e). Consider

$$
\begin{aligned}
\int_{0}^{T} G^{\prime}(t, s) d s & =-\frac{1}{2} \int_{0}^{t} s^{\alpha-2} d s+\frac{1}{2} \int_{t}^{T} s^{\alpha-2} d s \\
& =-\frac{1}{2}\left[\frac{t^{\alpha-1}}{\alpha-1}\right]+\frac{1}{2}\left[\frac{T^{\alpha-1}}{\alpha-1}-\frac{t^{\alpha-1}}{\alpha-1}\right] \\
& \leq \frac{T^{\alpha-1}}{2(\alpha-1)}
\end{aligned}
$$

This completes the proof of $(\mathrm{f})$. For $(\mathrm{g})$, consider

$$
\int_{0}^{T} s^{2-\alpha} G^{\prime}(t, s) d s=-\frac{1}{2} \int_{0}^{t} d s+\frac{1}{2} \int_{t}^{T} d s=-\frac{t}{2}+\frac{T}{2}-\frac{t}{2}=\frac{T}{2}-t \leq \frac{T}{2} .
$$

Consider

$$
\begin{aligned}
\int_{0}^{T}|G(t, s)| d s= & \int_{0}^{t}\left|G_{1}(t, s)\right| d s+\int_{t}^{T}\left|G_{2}(t, s)\right| d s \\
\leq & \int_{0}^{t}\left(\frac{T}{4}+\left|\frac{s-t}{2}\right|\right) s^{\alpha-2} d s+\int_{t}^{T}\left(\frac{T}{4}+\left|\frac{t-s}{2}\right|\right) s^{\alpha-2} d s \\
= & \frac{T t^{\alpha-1}}{4(\alpha-1)}-\int_{0}^{t}\left(\frac{s-t}{2}\right) s^{\alpha-2} d s+\frac{T}{4}\left(\frac{T^{\alpha-1}}{\alpha-1}-\frac{t^{\alpha-1}}{\alpha-1}\right) \\
& -\int_{t}^{T}\left(\frac{t-s}{2}\right) s^{\alpha-2} d s \\
= & \frac{T t^{\alpha-1}}{4(\alpha-1)}-\frac{t^{\alpha}}{2 \alpha}+\frac{t^{\alpha}}{2(\alpha-1)}-\frac{t\left(T^{\alpha-1}-t^{\alpha-1}\right)}{2(\alpha-1)}+\frac{T^{\alpha-1}-t^{\alpha-1}}{2 \alpha}
\end{aligned}
$$




$$
\leq \frac{T^{\alpha}}{4(\alpha-1)}+\frac{T^{\alpha}}{\alpha-1}+\frac{T^{\alpha}}{2 \alpha}=\frac{T^{\alpha}(7 \alpha-2)}{4 \alpha(\alpha-1)}
$$

Thus, we have (h). The proof is complete.

We are now able to formulate a Lyapunov-type inequality for the anti-periodic boundary value problem.

Theorem 3.2. If (1.3) has a nontrivial solution, then

$$
\int_{0}^{T} s^{\alpha-2}|p(s)| d s \geq \frac{4}{T}
$$

Proof. Let $C[0, T]$ be the Banach space of continuous functions $y$ on $[0, T]$ with the norm

$$
\|y\|_{C}=\max _{t \in[0, T]}|y(t)|
$$

It follows from Theorem 3.1 that a solution to (1.3) satisfies the equation

$$
y(t)=\int_{0}^{T} G(t, s) p(s) y(s) d s .
$$

Consider

$$
\begin{aligned}
|y(t)| & =\left|\int_{0}^{T} G(t, s) p(s) y(s) d s\right| \\
& \leq \int_{0}^{T}|G(t, s)||p(s)||y(s)| d s \\
& \leq\|y\| \int_{0}^{T}|G(t, s)||p(s)| d s \\
& =\|y\| \int_{0}^{T}\left[s^{2-\alpha}|G(t, s)|\right]\left|s^{\alpha-2} p(s)\right| d s
\end{aligned}
$$

which implies

$$
\|y\| \leq\|y\| \max _{s \in[0, T]}\left[s^{2-\alpha}|G(t, s)|\right]\left[\int_{0}^{T}\left|s^{\alpha-2} p(s)\right| d s\right] .
$$

An application of Lemma 3.1 yields the result. The proof is complete.

\section{Application}

In this section, we estimate a lower bound for the eigenvalue of the conformable eigenvalue problem corresponding to the conformable boundary value problem (1.3) using three different methods.

Definition 4.1 ([17]). A Lyapunov Inequality Lower Bound (LILB) is defined as a lower estimate for the smallest eigenvalue obtained from Lyapunov-type inequality given in (3.15) by setting $p(s)=\lambda$, that is,

where $G_{\max }=\max _{0 \leq t \leq T}|G(t, s)|$.

$$
\lambda \geq \frac{1}{T G_{\max }},
$$


Definition 4.2 ([17]). A Cauchy-Schwartz Inequality Lower Bound (CSILB) is defined as a lower bound for the smallest eigenvalue obtained from Cauchy-Schwartz inequality of type given in (3.15) by setting $p(s)=\lambda$, that is,

$$
\lambda \geq\left[\int_{0}^{T} \int_{0}^{T} G^{2}(t, s) d s d t\right]^{-\frac{1}{2}} .
$$

Definition 4.3 ([17]). A Semi Maximum Norm Lower Bound (SMNLB) is defined as a lower bound for the smallest eigenvalue obtained from Semi Maximum Norm inequality of type given in given in (3.15) by setting $p(s)=\lambda$, that is,

$$
\lambda \geq \frac{1}{\max _{0 \leq t \leq T} \int_{0}^{T}|G(t, s)| d s} .
$$

Theorem 4.1. Assume that $y$ is a nontrivial solution of the conformable eigenvalue problem

$$
\left\{\begin{array}{l}
\left(T_{0+}^{\alpha} y\right)(t)+\lambda y(t)=0, \quad 0<t<T \\
y(0)+y(T)=0, \quad y^{\prime}(0)+y^{\prime}(T)=0
\end{array}\right.
$$

where $y(t) \neq 0$ for each $t \in(0, T)$. Then

$$
\begin{gathered}
\lambda_{(L I L B)} \geq \frac{4(\alpha-1)}{T^{\alpha}}, \quad 1<\alpha \leq 2, \\
\lambda_{(C S I L B)} \geq \frac{4 \sqrt{(2 \alpha-3)}}{T^{\alpha}}, \quad \frac{3}{2} \leq \alpha \leq 2, \\
\lambda_{(S M N L B)} \geq \frac{4 \alpha(\alpha-1)}{T^{\alpha}(7 \alpha-2)}, \quad 1<\alpha \leq 2 .
\end{gathered}
$$

Proof. We choose $p(s)=\lambda$ in (3.15). Then, we obtain,

$$
\lambda \int_{0}^{T} s^{\alpha-2} d s \geq \frac{4}{T}
$$

implies

$$
\lambda\left(\frac{T^{\alpha-1}}{\alpha-1}\right) \geq \frac{4}{T}
$$

This proves the result (4.2). Consider,

$$
\begin{aligned}
\lambda & \geq\left[\int_{0}^{T} \int_{0}^{T} G^{2}(t, s) d s d t\right]^{-\frac{1}{2}} \\
& =\left(\int_{0}^{T} \int_{0}^{T}\left|s^{2-\alpha} G(t, s)\right|^{2} s^{2 \alpha-4} d s d t\right)^{-\frac{1}{2}} \\
& \geq\left(\frac{T^{2}}{16} \int_{0}^{T} \int_{0}^{T} s^{2 \alpha-4} d s d t\right)^{-\frac{1}{2}}
\end{aligned}
$$




$$
=\frac{4}{T}\left(\frac{T^{2 \alpha-2}}{2 \alpha-3}\right)^{-\frac{1}{2}}=\frac{4 \sqrt{(2 \alpha-3)}}{T^{\alpha}} .
$$

So, (4.3) is proved. The result (4.4) follows from (4.1) and (3.14). The proof is complete.

\section{REFERENCES}

[1] T. Abdeljawad, On conformable fractional calculus, J. Comput. Appl. Math. 279 (2015), 57-66.

[2] T. Abdeljawad, J. Alzabut and F. Jarad, A generalized Lyapunov-type inequality in the frame of conformable derivatives, Adv. Difference Equ. 2017(321) (2017), 10 pages.

[3] D. R. Anderson, Positive Green's functions for boundary value problems with conformable derivatives, in: T. M. Rassias and V. Gupta (Eds.), Mathematical Analysis, Approximation Theory and Their Applications, Springer, Basel, 2016, 63-74.

[4] D. R. Anderson and R. I. Avery, Fractional-order boundary value problem with Sturm-Liouville boundary conditions, Electron. J. Differential Equations 2017(29) (2017), 10 pages.

[5] D. R. Anderson, E. Camrud and D. J. Ulness, On the nature of the conformable derivative and its applications to physics, J. Fract. Calc. Appl. 10(2) (2019), 92-135.

[6] D. R. Anderson and D. J. Ulness, Properties of the Katugampola fractional derivative with potential application in quantum mechanics, J. Math. Phys. 56(6) (2015), 18 pages.

[7] A. Atangana, D. Baleanu and A. Alsaedi, New properties of conformable derivative, Open Math. 13 (2015), 889-898.

[8] R. C. Brown and D. B. Hinton, Lyapunov inequalities and their applications, in: T. M. Rassias (Ed.), Survey on Classical Inequalities, Math. Appl. 517, Springer and Business Media, Dordrecht, 2000, 1-25.

[9] Y. Gholami and K. Ghanbari, Fractional Lyapunov inequalities on spherical shells, Differ. Equ. Appl. 9(3) (2017), 353-368.

[10] F. Jarad, E. Ugurlu, T. Abdeljawad and D. Baleanu, On a new class of fractional operators, Adv. Difference Equ. 2017(247) (2017), 16 pages.

[11] U. N. Katugampola, A new approach to generalized fractional derivatives, Bull. Math. Anal. Appl. 6(4) (2014), 1-15.

[12] R. Khalil, M. Al Horani, A. Yousef and M. Sababheh, A new definition of fractional derivative, J. Comput. Appl. Math. 264 (2014), 65-70.

[13] A. A. Kilbas, H. M. Srivastava and J. J. Trujillo, Theory and Applications of Fractional Differential Equations, Elsevier, Amsterdam, 2006.

[14] A. Liapounoff, Problème général de la stabilité du mouvement, Ann. Fac. Sci. Toulouse Math. 9 (1907), 203-474.

[15] M. D. Ortigueira and J. A. T Machado, What is a fractional derivative?, J. Comput. Phys. 293 (2015), 4-13.

[16] B. G. Pachpatte, On Lyapunov-type inequalities for certain higher order differential equations, J. Math. Anal. Appl. 195(2) (1995), 527-536.

[17] N. Pathak, Lyapunov-type inequality and eigenvalue estimates for fractional problems, Ph.D. Thesis, Southern Illinois University, Carbondale, 2016.

[18] J. P. Pinasco, Lyapunov-type Inequalities With Applications to Eigenvalue Problems, Springer, New York, 2013.

[19] I. Podlubny, Fractional Differential Equations, Academic Press Inc., San Diego, 1999.

[20] V. E. Tarasov, No nonlocality. No fractional derivative, Commun. Nonlinear Sci. Numer. Simul. 62 (2018), 157-163.

[21] A. Tiryaki, Recent developments of Lyapunov-type inequalities, Adv. Dyn. Syst. Appl. 5(2) (2010), 231-248. 
[22] X. Yang, Y. Kim and K. Lo, Lyapunov-type inequality for a class of even-order linear differential equations, Appl. Math. Comput. 245 (2014), 145-151.

[23] X. Yang, Y. Kim and K. Lo, Lyapunov-type inequalities for a class of higher-order linear differential equations, Appl. Math. Lett. 34 (2014), 86-89.

[24] D. Zhao and M. Luo, General conformable fractional derivative and its physical interpretation, Calcolo 54(3) (2017), 903-917.

${ }^{1}$ Department of Mathematics,

Birla Institute of Technology and Science Pilani, HYDERABAD - 500078, TElanganA, INDIA

Email address: j.jaganmohan@hotmail.com

${ }^{2}$ Department of Mathematics,

Birla Institute of Technology and Science Pilani,

Hyderabad - 500078, Telangana, India

Email address: debananda04@gmail.com

${ }^{3}$ Department of Mathematics,

Birla Institute of Technology and Science Pilani, HyderABAD - 500078, TElanganA, IndiA

Email address: dipak@hyderabad.bits-pilani.ac.in 